\title{
A percepção das enfermeiras acerca da sua atuação ante os direitos dos clientes
}

\author{
NURSES' PERCEPTION OF THEIR WORK VIS-A-VIS PATIENTS' RIGHTS
}

\author{
LA PERCEPCIÓN DE LAS ENFERMERAS RESPECTO A SU ACTUACIÓN \\ ANTE LOS DERECHOS DE LOS CLIENTES
}

\author{
Patrícia Chaves Alves ${ }^{1}$, Valéria Lerch Lunardi², \\ Guilherme Lerch Lunardi ${ }^{3}$, Wilson Danilo Lunardi Filho ${ }^{4}$
}

\section{RESUMO}

Com o objetivo de conhecer a percepção das enfermeiras acerca de sua atuação ante os direitos dos clientes e desenvolver um instrumento que possibilitasse mensurar esta percepção, realizou-se uma pesquisa exploratório-descritiva, com uma amostra de 73 enfermeiras de dois hospitais. Mediante um questionário autoaplicado, foram identificados e validados quatro construtos relacionados à atuação das enfermeiras, quanto aos direitos dos clientes: preservação da individualidade; respeito à autonomia do cliente, identificação pessoal e informação para a tomada de decisão. 0 construto que apresentou me-lhor desempenho diz respeito à preservação da individualidade. Já o respeito à autonomia do cliente e a identificação pessoal situaram-se em um nível intermediário, enquanto que o construto informação para a tomada de decisão apresentouse como o menos realizado pelas enfermeiras. A preservação da individualidade e 0 respeito à autonomia mostraram-se como os construtos que mais influenciam a per-cepção das enfermeiras de como, em geral, respeitam os direitos dos clientes.

\section{DESCRITORES}

Ética de enfermagem.

Direitos do paciente.

Autonomia pessoal.

Individualidade.

\section{ABSTRACT}

This exploratory-descriptive survey was carried out with a sample consisting of 73 nurses from two hospitals in order to know the perception they have of their work via-a-vis patients' rights and develop an instrument capable of measuring such perception. Four constructs referring to the nurses' performance via-a-vis patients' rights were identified and validated, based on questions the nurses answered: preservation of individuality; respect for the patient's autonomy; personal identification; and information for decision-taking. Preservation of individuality was the construct with the best performance. Respect for the patient's autonomy and personal identification appear in an intermediate level, whereas the construct information for decision-taking was the least used. In general, preservation of individuality and respect for the patient's autonomy are the constructs that influenced the most nurses' perception on patients' rights.

\section{KEY WORDS}

Ethics, nursing.

Patient rights.

Personal autonomy.

Individuality.

\section{RESUMEN}

Con el objetivo de conocer la percepción de las enfermeras referentes a su actuación ante los derechos de los clientes y para desarrollar un instrumento para mensurar esta percepción, se realizó una investigación exploratorio-descriptiva, con una muestra de 73 enfermeras de dos hospitales. Por medio de un cuestionario autoaplicado, fueron identificados y validados cuatro constructos relacionados a la actuación de las enfermeras, en cuanto a los derechos de los clientes: preservación de la individualidad, respeto a la autonomía del cliente, identificación personal e información para la toma de decisión. El constructo que presentó un mejor desempeño se refiere a la preservación de la individualidad. Ya el respeto a la autonomía del cliente y la identificación personal se situaron en un nivel intermedio, mientras que el constructo información para la toma de decisión se presentó como el menos realizado por las enfermeras. La preservación de la individualidad y el respecto a la autonomía se mostraron como los constructos que más influencian la percepción de las enfermeras de como, en general, respetan los derechos de los clientes.

\section{DESCRIPTORES}

Ética de enfermería.

Derechos del paciente.

Autonomía personal.

Individualidad.

\footnotetext{
${ }^{1}$ Enfermeira do Hospital São Lucas da Pontifícia Universidade Católica do Rio Grande do Sul. Porto Alegre, RS, Brasil. pacalves@yahoo.com.br 2 Enfermeira. Doutora em Enfermagem. Professora Associada do Departamento de Enfermagem da Fundação Universidade Federal do Rio Grande - FURG. Rio Grande, RS, Brasil. vlunardi@terra.com.br ${ }^{3}$ Administrador. Mestre em Administração. Professor Assistente do Departamento de Ciências Econômicas, Administrativas e Contábeis da FURG. Graduando do Curso de Doutorado do Programa de Pós-Graduação em Administração da Universidade Federal do Rio Grande do Sul - UFRGS. Rio Grande, RS, Brasil. gllunardi@ea.ufrgs.br ${ }^{4}$ Enfermeiro. Doutor em Enfermagem. Professor Associado do Departamento de Enfermagem da FURG. Rio Grande, RS, Brasil. lunardifilho@terra.com.br
} 


\section{INTRODUÇÃO}

Há décadas, a preocupação com a ética vem emergindo cada vez mais fortemente em nossa sociedade, mais precisamente no âmbito da saúde, pelas diversas modificações, descobertas e experimentos científicos. A enfermagem vem aprofundando suas reflexões e questionamentos sobre a sua prática cotidiana, a fim de enfrentar esses desafios, bem como as questões éticas que cotidianamente surgem. A busca de soluções para os muitos dilemas enfrentados na área da saúde necessita de um esforço coletivo dos profissionais que aí atuam, sendo que a equipe de enfermagem pode oferecer uma contribuição importante, já que são os profissionais que mais tempo permanecem com o cliente. Apesar de os direitos do cliente hospitalizado constituírem um tema já conhecido e preconizado na área da saúde, em nossa realidade, parece ainda estar sendo pouco valorizado $^{(1-2)}$ e investigado.

São direitos do cliente, dentre outros, receber um atendimento atencioso e respeitoso; a dignidade pessoal; 0 sigilo profissional; conhecer a identidade dos profissionais envolvidos em seu tratamento; obter informação clara, numa linguagem acessível, sobre o diagnóstico, tratamento e prognóstico; recusar tratamento e ser informado das possíveis conseqüências dessa atitude; reclamar sem sofrer qualquer represália(3-4).

A falta de comunicação entre as equipes e a necessidade de uma maior prioridade no uso do tempo das enfermeiras, no que se refere à orientação dos clientes, são importantes variáveis no seu cuidado ${ }^{(5-7)}$. Por vezes, nos deparamos com posturas autoritárias e até desrespeitosas por parte de alguns profissionais da saúde, frente aos direitos dos clientes, provocando vários questionamentos: Como as enfermeiras percebem estes direitos? As enfermeiras têm respeitado tais direitos?

Dessa forma, preocupados com o fazer da enfermagem e, principalmente, com 0 respeito aos direitos dos clientes, tivemos por objetivos:

- conhecer a percepção das enfermeiras acerca de sua atuação frente aos direitos dos clientes; e

- desenvolver um instrumento que possibilite mensurar a percepção das enfermeiras acerca de sua atuação frente aos direitos dos clientes.

\section{MÉTODO}

Trata-se de um estudo quantitativo de caráter exploratório-descritivo, que foi desenvolvido em 2005, em dois hospitais gerais do sul do país - um hospital universitário (A) e outro beneficente (B). A coleta de dados foi realizada através de questionários auto-administrados (contendo perguntas simples, acompanhadas de instruções detalhadas sobre o seu preenchimento e sobre a pesqui- sa). Os questionários foram entregues às 91 enfermeiras destas instituições, sendo 56 no Hospital A e 35 no B, retornando 73 questionários, caracterizando $80,2 \%$ da população alvo.

\section{Desenvolvimento do Instrumento}

Antecedentes da Pesquisa - Para a construção do questionário, utilizou-se fundamentalmente uma cartilha em que são arrolados diferentes direitos dos clientes ${ }^{(2)}$. 0 questionário foi composto por 16 questões fechadas (15 relacionadas ao respeito da enfermeira frente aos direitos do cliente e uma avaliando, de modo geral, se a profissional respeita estes direitos). 0 mesmo foi operacionalizado em uma escala tipo Likert de 5 pontos, variando de 1 para nunca a 5 para sempre, além de conter uma questão aberta, buscando investigar possíveis dificuldades para o cumprimento dos direitos dos clientes. Foram acrescentadas ao final do questionário, mais cinco questões de caracterização da amostra (idade, sexo, área de atuação, tempo de formado e tempo na instituição).

Validação do Instrumento - A validação do instrumento ocorreu em três momentos, através da: a) Validade de face: em que se verificou se as questões do instrumento apresentavam forma e vocabulário adequados ao propósito da mensuração; b) Validade de conteúdo: em que se verificou se os itens do questionário representavam o conteúdo que se desejava avaliar, realizado através de dois pré-testes e suas respectivas análises; e c) Validade de traço ou construto, que verifica a ligação entre a teoria e as medidas utilizadas no instrumento ${ }^{(8)}$.

Assim, para garantir a validade de construto do instrumento, dois testes estatísticos foram realizados: a análise fatorial e 0 alfa de Cronbach. A análise fatorial como uma estatística destina-se essencialmente à redução e sumarização dos dados, possibilitando a formação de grupos de variáveis associadas entre si. 0 teste alfa de Cronbach (ou coeficiente alfa) verifica a confiabilidadedo instrumento e se diferentes características de cada um destes grupos, medidas através de questões do instrumento ou de indicadores dispostos no questionário, são consistentes ${ }^{(8)}$. Este último teste foi realizado com a finalidade de comprovar a fidedignidade das escalas utilizadas, ou seja, avaliar se as questões incluídas no questionário realmente estavam medindo o seu grupo (construto) de indicadores.

Aspectos Éticos - Foi solicitada uma autorização por escrito à chefia de enfermagem das instituições hospitalares envolvidas, bem como o consentimento livre e esclarecido dos sujeitos participantes, informando o objetivo e a metodologia do trabalho, assegurando o anonimato de suas respostas, assim como o seu direito de não participarem do estudo. 0 projeto foi aprovado pelo Comitê de Ética em Pesquisa da Área da Saúde-FURG (Processo 23116.002726/2005-06). 
RESULTADOS

A Tabela 1 apresenta os dados demográficos da amostra estudada. Em relação às características dos sujeitos do estudo, destaca-se como relevante que $69,9 \%$ das enfermeiras tem idade superior a 30 anos, o que aponta para sua maturidade. Além disso, a média de anos de trabalho na enfermagem é de aproximadamente 10 anos $(9,78)$, portanto um tempo médio razoável, sendo significativo, ainda, que o tempo de atuação destas profissionais na sua instituição hospitalar é de 6,01 anos.

Tabela 1 - Características dos sujeitos do estudo - Rio Grande - 2007

\begin{tabular}{|c|c|c|}
\hline Características & $\mathbf{N}$ & $\%$ \\
\hline \multicolumn{3}{|l|}{ Sexo } \\
\hline Feminino & 73 & 100 \\
\hline \multicolumn{3}{|l|}{ Idade } \\
\hline $20-30$ & 22 & 30,1 \\
\hline $31-40$ & 32 & 43,8 \\
\hline $41-50$ & 18 & 24,7 \\
\hline $51+$ & 1 & 1,4 \\
\hline Tempo de trabalho na Profissão (anos) & $(\mathrm{M}=9,78)$ & \\
\hline Tempo que atua na Instituição (anos) & $(\mathrm{M}=6,01)$ & \\
\hline \multicolumn{3}{|l|}{ Tipo de hospital que trabalha } \\
\hline Beneficente & 27 & 36,7 \\
\hline Universitário & 46 & 63,3 \\
\hline
\end{tabular}

A terceira e última etapa do processo de elaboração e validação do instrumento desenvolvido - a validade de construto - foi realizada logo após a aplicação dos questionários. A análise fatorial agrupou as questões referentes ao respeito da enfermeira quanto aos direitos dos clientes em quatro diferentes dimensões (construtos), representando diferentes facetas percebidas quanto aos seus direitos. A análise sugeriu ainda a eliminação de três questões do questionário, por não apresentarem coerência conceitual com as dimensões encontradas ou por apresentarem cargas fatoriais elevadas (superiores a 0,40) em mais de um dos fatores. As quatro dimensões propostas explicam 73,6\% da variação das questões originais, o que representa um elevado grau de sintetização dos dados - facilitando, portanto, seu manuseio e interpretação. A Tabela 2 apresenta as cargas fatoriais de cada construto, de acordo com sua formação nos fatores, os quais correspondem às quatro dimensões ${ }^{(a)}$ a serem discutidas nos resultados.

Tabela 2 - Análise Fatorial Exploratória (rotação Varimax) - Rio Grande - 2007

\begin{tabular}{|c|c|c|c|c|}
\hline Indicadores & F1 & F2 & F3 & F4 \\
\hline \multicolumn{5}{|l|}{ Informação para a tomada de decisão } \\
\hline Forneço ao cliente informações compreensíveis sobre o nome dos medicamentos administrados. & ,789 & & & \\
\hline $\begin{array}{l}\text { Forneço ao cliente informações compreensíveis sobre os possíveis efeitos dos medicamentos } \\
\text { administrados. }\end{array}$ & ,908 & & & \\
\hline $\begin{array}{l}\text { Forneço ao cliente informações compreensíveis sobre possíveis riscos das ações diagnósticas durante a } \\
\text { sua internação hospitalar. }\end{array}$ & ,908 & & & \\
\hline $\begin{array}{l}\text { Forneço ao cliente informações compreensíveis sobre possíveis riscos da terapêutica instituída durante a } \\
\text { sua internação hospitalar. }\end{array}$ & ,905 & & & \\
\hline $\begin{array}{l}\text { Forneço ao cliente informações compreensíveis sobre possíveis benefícios da terapêutica instituída } \\
\text { durante a sua internação hospitalar. }\end{array}$ & ,876 & & & \\
\hline \multicolumn{5}{|l|}{ Respeito à autonomia do cliente } \\
\hline Respeito o direito do cliente de consentir ou recusar procedimentos de enfermagem nele realizados. & & ,891 & & \\
\hline Respeito o direito do cliente de consentir ou recusar ações terapêuticas a que será submetido. & & ,924 & & \\
\hline Respeito o direito do cliente de ter acesso a seu prontuário. & &, 592 & & \\
\hline \multicolumn{5}{|l|}{ Preservação da individualidade } \\
\hline Respeito o pudor e intimidade do meu cliente. & & & ,833 & \\
\hline Asseguro o sigilo de toda e qualquer informação pessoal do meu cliente. & & & ,761 & \\
\hline Respeito o meu cliente de qualquer discriminação. & & & ,669 & \\
\hline \multicolumn{5}{|l|}{ Identificação pessoal } \\
\hline $\begin{array}{l}\text { Identifico-me ao cliente que está sob meus cuidados através de uma apresentação prévia (informando o } \\
\text { meu nome, cargo e função). }\end{array}$ & & & &, 741 \\
\hline Chamo o cliente pelo nome e sobrenome. & & & &, 836 \\
\hline Initial eigenvalue & 4,42 & 2,28 & 1,73 & 1,15 \\
\hline \% variância explicada - rotated $(73,6 \%)$ & $34,0 \%$ & $17,5 \%$ & $13,3 \%$ & $8,8 \%$ \\
\hline Alfa de Cronbach & 0,92 & 0,72 & 0,63 & 0,54 \\
\hline KMO medida de adequação da amostra $(\mathrm{KMO}=0,74)$ & & & & \\
\hline Teste de Bartlet: qui-quadrado $=418,064$ & & & & \\
\hline
\end{tabular}

(a) Fatores, dimensões e construtos são utilizados com o mesmo sentido conceitual.

Rev Esc Enferm USP
$2008 ; 42(2): 242-8$.
www.ee.usp.br/reeuspl $\begin{aligned} & \text { A percepção das enfermeiras acerca da sua } \\ & \text { atuação ante aos direitos dos clientes }\end{aligned}$


A fidedignidade das quatro dimensões identificadas foi testada através do cálculo do alfa de Cronbach. Sugerem-se valores entre 0,60 e 0,80 para estudos exploratórios, garantindo dessa forma a confiabilidade das escalas utilizadas no instrumento ${ }^{(9)}$. Cabe lembrar que, quanto maior for 0 alfa, que varia de 0 a 1, maior será a fidedignidade das escalas. 0 alfa de Cronbach do instrumento apresentou valor 0,82 , enquanto os coeficientes das quatro dimensões situaram-se entre 0,54 e 0,92, o que confirma a fidedignidade de três das quatro dimensões identificadas (Tabela 2). Embora o coeficiente alfa do construto identificação pessoal $(0,54)$ tenha apresentado valor abaixo do indicado pela literatura, optou-se aqui pela sua manutenção, tendo em vista que o presente estudo apresenta características de uma pesquisa exploratória, exigindo, todavia , cuidados quanto à sua interpretação.

Dessa forma, os quatro construtos propostos foram definidos conceitualmente como: preservação da individualidade, compreendendo todas as ações implementadas para assegurar o respeito à intimidade e às características individuais do cliente; respeito à autonomia do cliente, que implica o direito do cliente autodeterminar-se nas questões que dizem respeito a si; identificação pessoal, referindo-se ao direito do cliente de tanto ser identificado individualmente quanto de poder identificar individualmente os profissionais com quem se relaciona; e informação para a tomada de decisão, como o direito do cliente de ser informado, de modo a subsidiar as tomadas de decisões.

Os demais resultados foram obtidos através de três diferentes análises estatísticas: 1) estatística descritiva, mediante as médias dos construtos e de seus indicadores, de modo a verificar quais os direitos que são mais (ou menos) respeitados pelas enfermeiras; 2 ) análise de regressão, buscando avaliar quais construtos têm maior efeito na percepção das enfermeiras acerca de seu respeito aos direitos dos clientes; e 3) análises de variância entre diferentes grupos de respondentes, para verificar se a percepção das enfermeiras acerca do respeito aos direitos dos clientes varia conforme elementos presentes na caracterização da amostra.

A análise descritiva permitiu identificar as percepções das enfermeiras acerca de sua atuação frente aos direitos dos clientes. Cada um dos quatro construtos identificados na pesquisa (preservação da individualidade, respeito à autonomia do cliente, identificação pessoal e informação para a tomada de decisão) foi operacionalizado através de um valor numérico, que representa a média aritmética das questões que o compuseram individualmente - agrupados previamente pela análise fatorial. As médias dos construtos e de seus respectivos indicadores são apresentados na Tabela 3.

Tabela 3 - Percepção quanto aos direitos do cliente respeitados pelas enfermeiras - Rio Grande - 2007

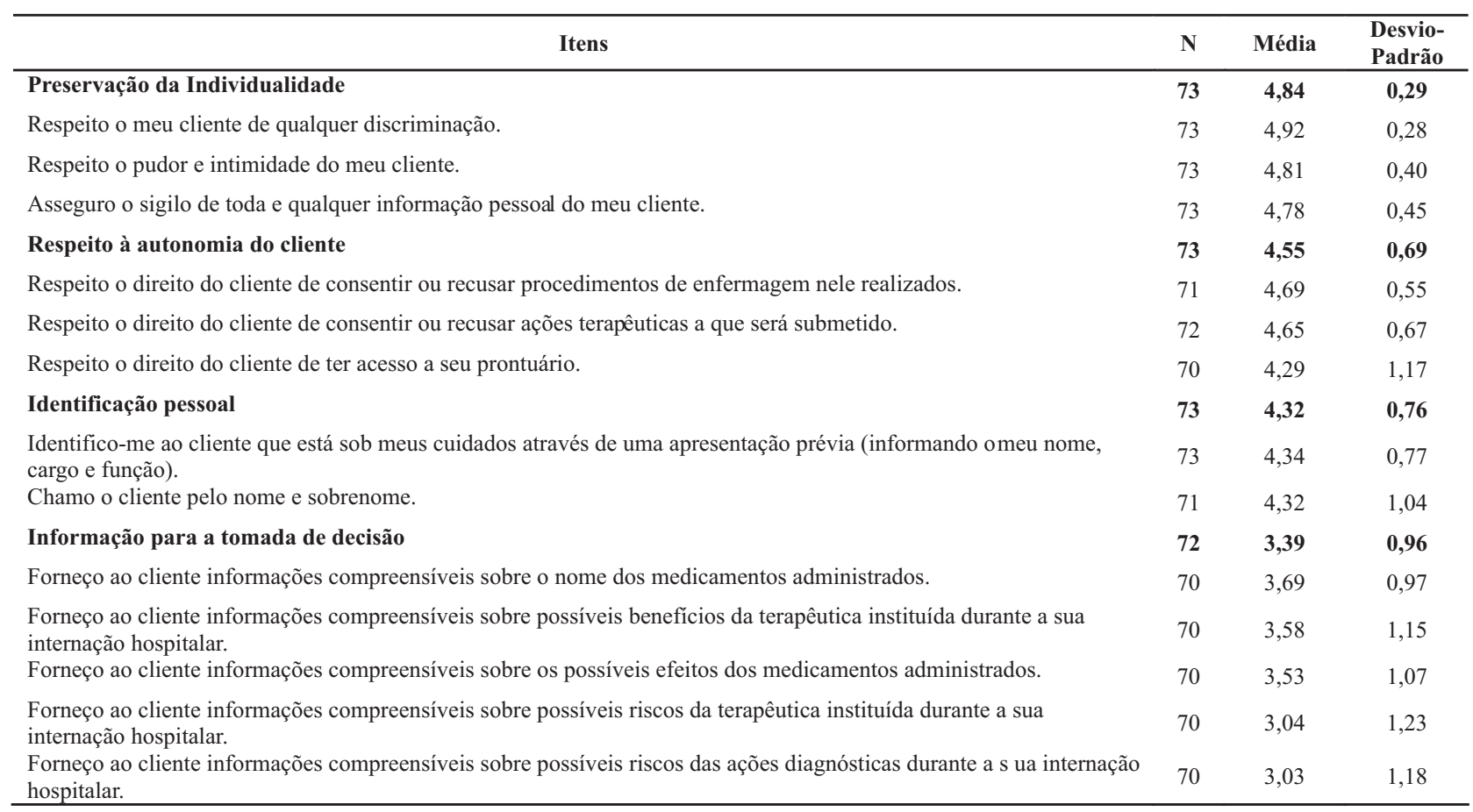

Os direitos dos clientes mais respeitados pelas enfermeiras investigadas dizem respeito à preservação da individualidade $(4,84)$. Num segundo grupo, com médias também bastante elevadas, aparecem os direitos relativos ao respeito à autonomia do cliente $(4,55)$ e à identificação pes- soal $(4,32)$. No entanto, pode-se perceber que os direitos dos clientes referentes à informação para a tomada de decisão apresentaram média bastante inferior aos demais construtos avaliados, sendo muito próximo do ponto médio da escala $(3,39)$. 
Na percepção das enfermeiras investigadas, os direitos que elas mais respeitam são os relacionados a evitar qualquer tipo de discriminação $(4,92)$; à preservação do pudor e intimidade do cliente $(4,81)$; e ao sigilo de toda e qualquer informação pessoal $(4,78)$. A preservação da individualidade, um dos princípios fundamentais da enfermagem e um direito moral do cliente $^{(10)}$, é percebida pelas enfermeiras como o grupo de direitos dos clientes que mais respeitam na sua prática profissional - dentre os construtos identificados.

No que se refere à Identificação pessoal, as enfermeiras percebem que, na grande maioria das vezes, se identificam ao cliente $(4,33)$ - 0 cliente tem direito de saber quem são os responsáveis por seus cuidados, seja através de crachás ou mesmo de uma apresentação prévia do cuidador, informando o seu cargo e função; como também afirmam identificar muito freqüentemente 0 cliente pelo nome $(4,32)$, o que o caracteriza como ser único e que deve ser tratado como tal, livre de qualquer forma que possa parecer desrespeitosa e/ ou preconceituosa como no uso de códigos, números ou apelidos. Portanto, identificação e apresentação prévias mostram-se fundamentais, pois como falar em direitos dos clientes e com eles produzir relações terapêuticas e de cuidado, sem previamente nos apresentarmos, identificando nossos nomes, demonstrando ter conhecimento de quem somos e que papéis e atribuições podem ser desempenhados em nossas interações?

No que se refere à autonomia, as enfermeiras percebem respeitar com grande freqüência o direito do cliente de consentir ou recusar procedimentos de enfermagem nele realizado $(4,69)$; além do direito de consentir ou recusar ações terapêuticas a que será submetido $(4,65)$ e de ter acesso a seu prontuário $(4,29)$. Para falar da autonomia do cliente, é imprescindível uma melhor compreensão do ser humano e seus direitos na sociedade. A Autonomia, do grego autos - eu e nomos - lei, é a capacidade especificamente humana de agir de acordo com sua vontade, através de escolhas que estão ao seu alcance, diante de objetivos por ela estabelecidos ${ }^{(11)}$. Desse modo, pode ter o significado de autogo-verno, direitos de liberdade, privacidade, escolha individual, liberdade da vontade, ser o motor do próprio comportamento e pertencer a si mes$m o^{(12)}$. Em outras palavras, autonomia é a capacidade de decidir por si mesmo nas questões que dizem respeito a si próprio como indivíduo, ou seja, como um sujeito capaz de deliberar sobre suas escolhas pessoais, devendo ser tratado com respeito à sua capacidade de autodeterminação.

$\mathrm{Na}$ área da saúde, reconhecer que os indivíduos são seres livres e autônomos para determinarem seu próprio destino implica oferecer alternativas terapêuticas e de cuidado, explicitar os riscos e benefícios inerentes a cada uma delas, certificar-se de que os clientes tenham compreendido claramente todas as informações prestadas e, por fim, respeitar sua decisão final. 0 mesmo se aplica àqueles que, de alguma maneira, estão impedidos de decidir sobre si, como as crianças ou aqueles em coma, cabendo ao profissional prestar informações claras aos seus responsáveis, respeitando as decisões que forem por eles tomadas( ${ }^{(4)}$.

Esta forma de agir contrapõe-se ao paternalismo, em que a decisão sobre a conduta não deriva do livre arbítrio dos clientes ou seus responsáveis. A posição paternalista origina-se nas normas hipocráticas, segundo as quais 0 conhecimento e a razão do profissional deveriam orientar a melhor conduta terapêutica, independentemente da vontade dos clientes. 0 conceito de consentimento informado é ainda recente no campo da ética em saúde, distinto do princípio que se baseia no juramento de Hipócrates não causar dano e sedar a dor. Na época de Hipócrates, a autonomia do cliente era dispensável, pois a arte de curar tinha como pressuposto 0 paternalismo ${ }^{(13)}$.

A declaração de vontade do cliente ocorre pelo seu consentimento informado, que é a sua manifestação, quando capaz, de tomar decisões quanto à sua saúde, após ter recebido a informação necessária sobre procedimentos terapêuticos, terapias alternativas, numa linguagem acessível, completa, entendendo e decidindo, sem ter sido submetido à coação, influência, indução ou intimidação, por parte de qualquer pessoa.

Por fim, as enfermeiras questionadas informaram que fornecem, ainda que menos frequentemente, informações compreensíveis sobre o nome dos medicamentos administrados $(3,69)$, seus possíveis efeitos $(3,53)$, possíveis riscos das ações diagnósticas $(3,03)$, possíveis riscos da terapêutica instituída $(3,04)$ e possíveis benefícios da terapêutica instituída, durante a sua internação hospitalar (3,52). A informação é um instrumento neces-sário e fundamental para que o cliente possa consentir ou recusar-se a medidas ou procedimentos de saúde a ele propostos ${ }^{(10)}$. Assim, todo e qualquer procedimento necessita ser realizado com o consentimento livre e esclarecido do cliente, a partir da informação e esclarecimento do tipo de medicamento e/ou procedimento, indicação, riscos e benefícios e do modo como será realizado, dentre outros. Ainda, mesmo que a responsabilidade sobre 0 diagnóstico e a terapêutica instituída seja de outro profissional da equipe de saúde, as enfermeiras necessitam esclarecer os clientes acerca dos possíveis procedimentos neles realizados, seus riscos e benefícios, de modo a que, informados, sejam estimulados a optar e se manifestar pela sua aceitação ou não. Informar os clientes, de modo claro e simples, acerca dos possíveis medicamentos a serem administrados não pode constituir-se apenas em uma opção da enfermeira, mas em uma obrigação, considerando-se 0 respeito aos direitos dos clientes ${ }^{(4)}$. 
Neste sentido, parece relevante destacar que, para um cliente expressar seu consentimento informado como expressão de sua autonomia, há que considerar o componente de informação, ou seja, a revelação da informação e sua compreensão do que é informado; e o componente de consentimento. Os seguintes elementos são entendidos como fundamentais em um consentimento informado:

Competência; Revelação; Entendimento; Voluntariedade; e Consentimento. [...] Um indivíduo dá um consentimento informado para uma intervenção se (e, talvez, somente se) for capaz de agir, receber uma exposição completa, entender a exposição, agir voluntariamente e consentir na intervenção ${ }^{(12)}$.

A segunda análise estatística realizada buscou identificar os construtos que mais influenciam a percepção das enfermeiras acerca da sua atuação geral, quanto ao respeito dos direitos dos clientes. Foi utilizado o modelo de regressão múltipla, verificando a relação de dependência existente entre os quatro construtos (variáveis independentes) e a questão que avalia, de modo geral, o respeito da enfermeira aos direitos dos clientes (variável dependente). Os resultados referentes a este teste apresentaram baixo grau de explicação $\left(r^{2}=0,203\right)$, demonstrando que a relação de causa-efeito explicada por estes quatro construtos é pequena, ou seja, existem outros fatores - não incluídos no instrumento de pesquisa - que impactam sobre a percepção geral da enfermeira quanto ao seu respeito pelos direitos do cliente, podendo estar relacionados à cultura organizacional, à hierarquia administrativa, normas organizacionais, priorização do tempo, dentre outros.

Entretanto, este teste possibilitou visualizar aspectos que influenciam a percepção das próprias enfermeiras, quanto a sua atuação, no que diz respeito aos direitos dos clientes (Tabela 4). Constatou-se que somente os construtos Preservação da individualidade e Respeito à autonomia se mostraram estatisticamente significativos $(p<0,05)$, sendo, portanto, as facetas que mais influenciam a percepção das enfermeiras de como, em geral, respeitam os direitos dos clientes. Estes achados demonstram que quanto mais os direitos relacionados à preservação da individualidade e à autonomia do cliente forem respeitados, maior será a percepção das enfermeiras de que estão respeitando os direitos dos clientes. Já os construtos Identificação pessoal e Informação para a tomada de decisão, na percepção das enfermeiras, não influenciam significativamente (em nível de $5 \%$ ) na sua avaliação geral, quanto a estarem ou não respeitando os direitos dos clientes.

Tabela 4 - Modelo de Regressão Múltipla - Rio Grande $-2007$

\begin{tabular}{lcc}
\hline \multicolumn{1}{c}{ Construtos } & Beta & $\boldsymbol{P}$ \\
\hline 1. Preservação da individualidade &, 235 & $\mathbf{, 0 3 8}$ \\
2. Respeito à autonomia do cliente &, 258 & $\mathbf{, 0 4 0}$ \\
3. Identificação pessoal &, 195 &, 102 \\
4. Informação para a tomada de decisão &,- 125 &, 283 \\
\hline
\end{tabular}

Uma questão relevante a ser ainda destacada referese ao construto Informação para a tomada de decisão como o menos realizado pelas enfermeiras, apesar da vinculação existente entre o respeito à autonomia do cliente e a sua necessidade de informação para a tomada de decisão, o que não parece ter sido confirmada através das percepções das enfermeiras. Porém, como respeitar a autonomia do cliente sem simultaneamente reconhecer 0 seu direito à informação para possibilitar uma tomada de decisões autônomas?

Para finalizar, após a análise de regressão, foram realizadas algumas análises de variância entre subgrupos da amostra (instituição, tempo de formado, tempo de trabaIho na instituição e idade). No entanto, a única variável que se mostrou estatisticamente significativa (em nível de 5\%) foi a instituição de origem dos profissionais (Tabela 5).

A diferença quanto à significância estatística $(p<0,05)$ indicou que as enfermeiras das duas instituições têm percepções distintas apenas quanto aos direitos relacionados à autonomia do cliente. Nos demais construtos, embora tenham apresentado médias ligeiramente diferentes, estas não se mostraram significantes estatisticamente.

Tabela 5 - Análise de variância entre as diferentes instituições - Rio Grande - 2007

\begin{tabular}{lccc}
\hline \multicolumn{1}{c}{ Construtos } & Hospital A & Hospital B & P \\
\hline Preservação da individualidade & 4,82 & 4,86 &, 522 \\
Respeito à autonomia do cliente & 4,69 & 4,30 &, $\mathbf{0 2 0}$ \\
Identificação pessoal & 4,27 & 4,40 &, 466 \\
Informação para a tomada de decisão & 3,53 & 3,16 &, 120 \\
\hline
\end{tabular}

As enfermeiras do hospital A, possivelmente por se tratar de um hospital universitário, percebem respeitar com maior freqüência o direito de o cliente consentir ou recusar procedimentos de enfermagem propostos, bem como ações terapêuticas a que será submetido, diferentemente da outra instituição. Outra diferença a ser destacada no estudo com relação aos profissionais das duas instituições investigadas foi que as enfermeiras do hospital $B$ (beneficiente) referem que se preocupam mais com questões relacionadas à identificação pessoal $(4,40)$, tanto dos profissionais que estão envolvidos no cuidado do cliente quanto na identificação do cliente, do que com 0 respeito à sua autonomia $(4,30)$; diferentemente do hospital $A$, cujas enfermeiras priorizam o respeito à autonomia (4,69 e 4,27, respectivamente). Este achado poderia ser justificado pelo programa de qualidade total incorporado ao Hospital B, o qual tem como uma das suas premissas básicas o uso de crachás e a apresentação prévia a todos os clientes ali atendidos.

\section{CONSIDERAÇÕES FINAIS}

Com este estudo, constatou-se que as enfermeiras perceberam os direitos dos clientes sob a ótica de quatro grandes grupos: Preservação da individualidade, Respeito à autonomia do cliente, Identificação pessoal como parte 
da preservação da(s) individualidade(s) e a informação para o cliente como pré-condição para a sua tomada de decisão.

Os dados referentes à idade, sexo, área de atuação, tempo de formado e tempo na instituição não mostraram diferença estatística na análise dos resultados, não sendo mencionados, dessa forma, no estudo. Este fato surpreendeu, pois esperavam-se diferentes percepções entre uma enfermeira com menor tempo de atuação e outra com maior tempo ou aquela que está há mais tempo na instituição, possivelmente, com maior influência da cultura da organização.

Outro dado interessante foi em relação à inversão dos construtos nos diferentes hospitais. Enquanto no Hospital $A$, a prioridade é a preservação da individualidade, seguido pelo respeito à autonomia, identificação pessoal do cliente e a informação para a tomada de decisão, no Hospital B, a autonomia do cliente não parece ser tão prioritária, sendo preconizada a identificação pessoal, reforçando aspectos da política de qualidade total implantada pela instituição.

\section{REFERÊNCIAS}

1. Clotet J. O consentimento informado nos Comitês de Ética em Pesquisa e na prática médica: conceituação, origens e atualidade. Bioética. 1995;3(1):51-9.

2. Santos LR, Beneri RL, Lunardi VL. Questões éticas no trabalho da equipe de saúde: 0 (des)respeito aos direitos do cliente. Rev Gaúcha Enferm. 2005;26(3):403-13.

3. Gauderer EC. Os direitos do cliente: um manual de sobrevivência. 6a ed. Rio de Janeiro: Record; 1998.

4. Conselho Federal de Enfermagem (COFEN). Resolução COFEN 311/2007. Aprova a reformulação do Código de Ética dos Profissionais de Enfermagem [legislação na Internet]. Brasília; 2007. [citado 2007 set. 5]. Disponível em: http:// www.notadez.com.br/content/normas. asp? $i d=36907$

5. Soares NV. A problematização dos direitos do cliente como desencadeadora da problematização dos direitos dos profissionais de enfermagem [dissertação]. Florianópolis: Programa de Pós-Graduação em Enfermagem, Universidade Federal de Santa Catarina; 2000.

6. Chaves $P$, Lunardi V, Costa V. A enfermagem frente aos direitos de clientes hospitalizados. Texto Contexto Enferm. 2005;14(1):38-43.
Como qualquer pesquisa científica, alguns cuidados devem ser levados em consideração, quando da interpretação dos resultados obtidos. 0 primeiro aspecto se refere à amostra analisada que, embora seja representativa em termos estatísticos (quanto ao número de respondentes), não permite que os resultados aqui encontrados sejam generalizados a outros contextos, que não as instituições estudadas. Sugere-se, ainda, que outros direitos referentes à identificação pessoal - construto que se mostrou mais frágil no instrumento - sejam inseridos e, posteriormente, validados estatisticamente no instrumento. Para pesquisas futuras, propõe-se a reutilização deste instrumento em outras instituições, bem como o redirecionamento de seu foco, por exemplo, para a percepção dos clientes acerca do respeito aos seus direitos pelo profissional de saúde, pois, para avaliar se seus direitos estão sendo respeitados e se o cuidado que lhes é prestado é qualificado, nada melhor que os próprios clientes para fazer essa avaliação.

7. Hoga LAK. A dimensão subjetiva do profissional na humanização da assistência à saúde: uma reflexão. Rev Esc Enferm USP. 2004;38(1):13-20.

8. Malhotra N. Pesquisa de marketing: uma orientação aplicada. 3a ed. Porto Alegre: Bookman; 2001.

9. Kotler P, Armstrong G. Princípios de marketing. Rio de Janeiro: Prentice-Hall do Brasil; 1993.

10. Fortes PAC. Ética e saúde: questões éticas, deontológicas e legais, tomada de decisões, autonomia e direitos do cliente, estudo de casos. São Paulo: EPU; 1998.

11. Selli L. Bioética na enfermagem. São Leopoldo: UNISI NOS; 1999.

12. Beauchamp TL, Childress JF. Princípios de ética biomédica. São Paulo: Loyola; 2002.

13. Clotet J, Goldim JR, Francisconi CF. Consentimento informado e a sua prática na assistência e pesquisa no Brasil. Porto Alegre: EDPUCRS; 2000. 